\section{Gen-Mutationen auch bei Metastasen noch prädiktiv}

\begin{abstract}
Mehrere Studien zeigten einen Zusammenhang zwischen Mutationen der Onkogene BRAF sowie NRAS und der Prognose von Melanomen in frühen Stadien. Ob diese Assoziationen auch für metastasierte Tumoren gelten, war die Frage einer aktuellen Studie.
\end{abstract}

D ie Arbeitsgruppe um John A. Jacobs wertete zunächst die Daten von insgesamt 677 Fällen Melanomen aus, bei denen eine Molekulardiagnostik der Onkogene BRAF (Exon 15) und NRAS (Exon 1 und 2) durchgeführt wurde.

Dabei zeigten sich Mutationen von BRAF in $47,3 \%$ sowie von NRAS in $20,1 \%$ der Fälle. In $94,4 \%$ lagen bei $B R A F-$ Mutationen Substitutionen von Valin durch Glutamin oder Lysin an Position 600 vor. Bei vier Patienten zeigten sich Mutationen beider Onkogene gleichzeitig. Die restlichen Testungen ergaben jeweils den Wildtyp der Onkogene.

Die Autoren analysierten die Daten von 519 Patienten, die ein Melanom im
Stadium IV entwickelt hatten. Studienteilnehmer mit einer BRAF- bzw. NRASMutation wiesen zu 24,4\% bzw. 21,3\% ZNS-Metastasen auf. Der Anteil von Patienten mit Wildtyp-Onkogenen und Hirnmetastasen lag dagegen nur bei $12,4 \%$ und damit signifikant niedriger. Umgekehrt waren Lungenmetastasen bei BRAF-Mutation $(55,2 \%)$ und NRAS-Mutation $(56,7 \%)$ signifikant seltener als bei Onkogenen vom Wildtyp (66,9\%). Für Leber-, Knochen-, Haut- oder Lymphknotenmetastasen ließen sich keine auffälligen Unterschiede feststellen.

Bei 313 Patienten mit Metastasierung (ohne Beteiligung der Uvea) war innerhalb von sechs Monaten nach Diagnose- stellung die Testung durchgeführt worden. Studienteilnehmer mit einer NRASMutation hatten mit einer medianen Überlebenszeit von 8,2 Monaten die schlechteste Prognose. Patienten mit Wildtyp-Onkogenen überlebten im Median 15,1 Monate. Patienten mit einer $B R A F-M u t a t i o n$ und einer entsprechenden zielgerichteten Therapie (Vemurafenib, Selumitinib) überlebten 15,6 Monate - signifikant länger als Patienten mit dieser Mutation ohne spezifische Therapie (10,3 Monate).

Die NRAS-Mutation war im Stadium IV ein unabhängiger Risikofaktor für ein früheres Versterben der Patienten.

Fazit: Mutationen der Onkogene $B R A F$ und NRAS waren in dieser Studie vermehrt mit ZNS-Metastasen assoziiert. Die Mutation von NRAS erwies sich darüber hinaus als unabhängiger negativer prognostischer Faktor.

Andreas Fischer

Jacobs JA et al. NRAS mutation status is an independent prognostic factor in metastatic melanoma. Cancer. 2012;118(16):4014-23.

\title{
Neue Kombinationstherapie bei fortgeschrittenem Melanom
}

\section{Die Prognose von Patienten mit metastasiertem Melanom bleibt nicht zuletzt aufgrund häufiger Hirnmetastasen äußerst schlecht. Ein Autorenteam prüfte in einer Phase-II-Studie den Einsatz von Ipilimumab kombinert mit dem Blut- Hirn-Schranke (BHS)-gängigen Fotemustin.}

A n der Studie nahmen Patienten mit inoperablem Tumor im Stadium III oder mit Tumorstadium IV teil. Zur Auswertung kamen die Daten von $86 \mathrm{~Pa}$ tienten, bei Studieneinschluss waren nur drei Teilnehmer ohne Metastasen. $20 \mathrm{~Pa}$ tienten wiesen asymptomatische Hirnmetastasen auf.

Die Patienten erhielten als Induktionstherapie Ipilimumab in einer Dosierung von $10 \mathrm{mg} / \mathrm{kg}$ alle drei Wochen (maximal vier Zyklen) sowie $100 \mathrm{mg}$ Fotemustin $/ \mathrm{m}^{2}$ dreimal in wöchentlichen Abständen. Fotemustin wurde dann im 21-Tage-Abstand von der neunten bis zur 24 . Woche weiter verabreicht.

Patienten mit einem klinischen Ansprechen auf diese Behandlung beka- men eine Erhaltungstherapie mit Ipilimumab alle zwölf Wochen sowie Fotemustin alle drei Wochen.

$34 \%$ der Studienteilnehmer erhielten mindestens einen Zyklus mit Ipilimumab sowie $72 \%$ mindestens einen Zyklus mit Fotemustin als Erhaltungstherapie. Insgesamt brachen im Studienverlauf $81 \%$ die Behandlung ab, $63 \%$ wegen Krankeitsprogression.

Bei 46,5\% der Patienten war eine definierte Immuntherapie-bedingte Tumorkontrolle möglich (19 Patienten mit partiellem Ansprechen, 15 Patienten mit stabiler Erkrankung, sechs Patienten mit kompletter Response). In der Untergruppe mit Hirnmetastasen war die Tumorkontrolle sogar in $50 \%$ der Fälle dokumentiert.
Die mediane Dauer bis zum Fortschreiten der Erkrankung lag bei 5,3 Monaten für das gesamte Studienkollektiv sowie bei 4,5 Monaten für Patienten mit Hirnmetastasen. Nach einem Jahr waren $53 \%$ aller Studienteilenehmer (54\% der Patienten mit Hirnmetastasen) noch am Leben

In 55\% der Fälle traten Grad-3- oder Grad-4-Reaktionen auf. Bei $24 \%$ der Patienten handelte es sich dabei um Thrombopenien sowie bei $19 \%$ um Neutropenien. Speziell auf die Immuntherapie bezogene häufigste Nebenwirkung von Grad 3 oder 4 waren Leberenzymerhöhungen mit $24 \%$.

Fazit: Die Autoren stufen die Kombination aus Ipilimumab und Fotemustin bei fortgeschrittenem Melanom inklusive Hirnmetastasen als effektiv ein. Allerdings fehlen hierzu noch Daten aus kontrollierten Studien. Andreas Fischer

Di Giacomo AM et al. Ipilimumab and fotemustine in patients with advanced melanoma (NIBITM1): an open label, single-arm phase 2 trial. Lancet Oncol. 2012;13(9):879-86. 